\title{
Numerical Simulation of a Contaminated Water Drop Sinking in a Oil by a Front-tracking Method*
}

\author{
Yasufumi YAMAMOTO**, Makoto YAMAUCHI ${ }^{* * *}$ and Tomomasa UEMURA** \\ ** Department of Mechanical Engineering, Kansai University \\ 3-35, Yamate-cho 3-chome, Suita, Osaka, 564-8680, Japan \\ E-mail: yamayasu@ kansai-u.ac.jp \\ *** Osaka Prefectural College of Technology
}

26-12, Saiwai-cho, Neyagawa, Osaka, 572-8572, Japan

\begin{abstract}
In this study, a front-tracking (FT) method combined with a solver for the interfacial transport of surfactant is proposed to analyze interfacial flows affected by contamination. Because the interfaces are expressed explicitly in the FT method, the advectiondiffusion equation on the interface can be treated easily and can be solved with high accuracy. In this study, a scheme that completely conserves the total amount of surfactant was constructed. Numerical simulations of a water drop sinking in silicone oil were performed, taking into account the Marangoni effect caused by concentrations. The effects of three parameters-a damping coefficient of interfacial tension, a diffusion coefficient, and the total amount of surfactant—were evaluated. Calculated results were compared with experimental results and were in very good agreement with variables including the stagnant cap size, the flow separation point, and the peak of shear stress. We can therefore expect that our simulations can estimate the conditions of surfactant at the interfaces.
\end{abstract}

Key words : Multiphase Flow, Numerical Analysis, Finite Difference Method, Surface Tension, Front-Tracking Method, Droplet, Contamination, Marangoni Effect

\section{Introduction}

The effects of contamination of interfaces in gas-liquid or liquid-liquid two-phase flow systems have been studied in various ways (e.g., Levich ${ }^{(1)}$ ), because the contamination not only reduces the terminal velocity but also affects the transport of mass, momentum, and energy between phases. Yamauchi, Ozawa and Uemura ${ }^{(2)}$ and Uemura and Yamauchi( ${ }^{(3)}$ developed the particle tracking velocimetry technique, which can simultaneously measure velocity fields on both sides of the interface of a water drop sinking in silicone oil, then evaluated the degree of contamination by the shearing condition on both sides of the interface. There are some numerical studies of the contamination, which are based on boundary-fitted coordinates calculations ${ }^{(4)-(8)}$. In their calculations, the inner flow of the gas bubble was neglected. However, because we are interested in liquid-liquid systems, in which the densities of both phases are of the same order, the inner flow of a liquid drop cannot be negligible.

Recently, some methods for solving multiphase flow problems using fixed rectangular grids, such as the volume-of-fluid (VOF) method ${ }^{(9)}$, the front-tracking method ${ }^{(10)}$, the level-set method $^{(11)}$, and the CIP method ${ }^{(12)}$ have been developed and are being widely used. James and Lowengrub $^{(13)}$ incorporated a model of surfactant transport on the interface in a VOF method and evaluated the effect of surfactant on the drop deformation. Xu and Zhang ${ }^{(14)}$ developed an Eulerian formulation of surfactant transport in the moving interface in the framework of the level-set method. In the VOF and level-set methods, however, interfaces are presented 
implicitly, so there are many difficulties in calculation of advection or spatial derivatives of concentration at the interface. On the other hand, in the front-tracking (FT) method, interfaces are explicitly represented, so advection and tangential gradients of concentrations can be calculated very easily and accurately. In addition, the effect of interfacial tension is given directly as the difference of tangential tensions, so the Marangoni effect caused by concentration gradient can be represented easily. Jan and Tryggvason ${ }^{(15)}$ performed FT simulations taking into account the interfacial advection of surfactant to examine the change of bubble rising velocity. In their model, neither diffusion nor adsorption/desorption processes were considered, so the concentration profile did not reach a steady state; therefore, their model cannot represent ac-

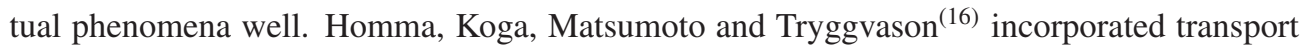
of solute in the bulk phase into their FT simulations, but calculation of the bulk concentration of solute was not directly incorporated to add to the benefits of the FT.

In this study, we propose a simulation method that uses an FT method combined with a solver of the transport of surfactant at the interface. We present a fully conservative scheme for the advection and diffusion of concentration on the interface, exploiting the characteristics of the FT method. The effects of the parameters of the surfactant transport are examined. We then try to reproduce the experimental results using contaminated drops as measured by Yamauchi et al. ${ }^{(2),(3)}$. In their experiments, the conditions of the surfactant are unknown. If we can find a set of parameters that allow the simulation to reproduce the experimentally observed results faithfully, we can use them to estimate the real surfactant conditions.

\section{Target of simulation}

As the target of simulations in this study, a water drop sinking in a silicone oil, as measured in detail by Yamauchi et al. ${ }^{(2)}$, was chosen. $100 \mathrm{cSt}$-silicone oil is placed in a $31 \mathrm{~mm}$ diameter cylindrical pipe. In the pipe, a water drop sinks by gravitational force. The physical properties of the fluids are shown in Table 1.

The diameter of the water drop is set to $d_{d}=15.4 \mathrm{~mm}$. The Reynolds number of the experimental result $R e=d_{d} U_{d} / v$ is 2.7 , where $U_{d}$ is the terminal velocity of the drop. Our simulations are performed using axisymmetric cylindrical coordinates because the flow fields observed in the experiments are axisymmetric. The calculated results are presented using polar coordinates $(r, \theta, \phi)$, with the origin at the center of the drop. To avoid confusion, the cylindrical coordinates are represented by $(x, \phi, z)$.

We do not have any information about the surfactant (e.g., physical properties, concentration) used in the experiments, so we do not know whether or not the adsorption-desorption process can be neglected. We therefore attempted simulations without the adsorption-desorption process as the first challenge of this study. We analyze the results at a fully developed state. Three parameters of the initial uniform concentration $\Gamma_{0}$, the diffusion coefficient $D_{s}$ on the interface (corresponding to the Schmidt number $S c_{s}=v / D_{s}$, where $v$ is the kinematic viscosity of the oil) and the damping coefficient $\alpha$ of interfacial tension (described later) are adjusted to reproduce the flow field observed in the experiment. From the calculated results, surfactant conditions are estimated.

\section{Numerical procedure}

\subsection{Front-tracking method}

The front-tracking (FT) method treats the multifluid system as one fluid with density and viscosity that vary spatially, and the interfacial tension effect acts as a delta function body force. In this section, we give a brief overview of the FT method (more detail is described in Ref. (17)).

One of the most characteristic features of the FT is that the interfaces are represented explicitly by connected marker points as front points. In FT, the basic equations are solved on a fixed grid system, with the assistance of the Lagrangian front points. The front points $\mathbf{x}_{s}$ are moved with a velocity interpolated from the velocities at the fixed grid points $\mathbf{x}$. An index 
function $I(\mathbf{x})$, which is an approximate Heaviside function smoothly varying at the interface from 0 to 1 , is defined on each fixed grid point, by using the front points' locations and normal vectors. Density $\rho(\mathbf{x})$ and viscosity $\mu(\mathbf{x})$ on the grid points are given by the linear function of the index function $I$ as:

$$
\begin{aligned}
& \rho(\mathbf{x})=I(\mathbf{x}) \rho_{\text {water }}+\{1-I(\mathbf{x})\} \rho_{\text {oil }}, \\
& \mu(\mathbf{x})=I(\mathbf{x}) \mu_{\text {water }}+\{1-I(\mathbf{x})\} \mu_{\text {oil }},
\end{aligned}
$$

where $I(\mathbf{x})=0$ in oil and $I(\mathbf{x})=1$ in water. The interfacial tension force $\mathbf{f}_{s}$ acting on each front element (line segment) can be calculated accurately by using tangent vectors at the front points, as described later. Conversion of information between front elements and fixed grid points is performed using a distribution function $D(\mathbf{x})$. For example, the front velocity $\mathbf{u}_{s}\left(\mathbf{x}_{s}\right)$ is obtained by:

$$
\mathbf{u}_{s}\left(\mathbf{x}_{s}\right)=\sum_{\mathbf{x}} D\left(\mathbf{x}-\mathbf{x}_{s}\right) \mathbf{u}(\mathbf{x}) \Delta x \Delta z
$$

and the converted body force $\mathbf{F}$ on fixed grid points is obtained from the surface force $\mathbf{f}_{s}$ by:

$$
\mathbf{F}(\mathbf{x})=\sum_{\mathbf{x}_{\mathbf{s}}} D\left(\mathbf{x}-\mathbf{x}_{s}\right) \mathbf{f}_{s}\left(\mathbf{x}_{\mathbf{s}}\right) \Delta s,
$$

where $\Delta s$ is the length of the element. The distribution function suggested by Peskin ${ }^{(18)}$ is used in this study:

$$
D(\mathbf{x})=h(x) h(z)
$$

where:

$$
h(r)=\left\{\begin{array}{ll}
\frac{1}{4 \Delta}\left(1+\cos \frac{\pi r}{2 \Delta}\right), & |r|<2 \Delta \\
0, & |r| \geq 2 \Delta
\end{array},\right.
$$

where $\Delta$ is the grid spacing. As a result of application of this function, the interface has a finite thickness of 4 grid units and the effect of the thickness is discussed later ( $\$ 4.1)$.

The nonuniform distribution of front marker points is avoided by adding or deleting them. In this study, the threshold $T_{a d d}$ for addition was empirically set to $T_{a d d}=0.8 \Delta x$, and the threshold $T_{d e l}$ for deletion was set to $T_{d e l}=0.6 \Delta x$. To interpolate the marker in the sparse region ( $\Delta s>T_{a d d}$ ), a 4-point Lagrangian polynomial was employed to represent the coordinates by the functions of arc length $s$ as $x_{s}=x_{s}(s), y_{s}=y_{s}(s)$. In the present study, in which drop shapes change little, this addition/deletion procedure does not have any bad influences upon the calculated flow field, because the marker interval is always smaller than the fixed-grid size and interpolation is done highly accurately. Tangent vectors are obtained by analytical derivatives of those functions as $(\mathrm{d} x / \mathrm{d} s, \mathrm{~d} y / \mathrm{d} s)$ and the normal vector as $(-\mathrm{d} y / \mathrm{d} s$, $\mathrm{d} x / \mathrm{d} s)$.

Fluids are assumed to be incompressible. The basic equations are as follows:

$$
\begin{aligned}
& \rho\left\{\frac{\partial \mathbf{u}}{\partial t}+\nabla \cdot \mathbf{u}\left(\mathbf{u}-\mathbf{u}_{g}\right)\right\}=-\nabla p+\nabla \cdot\left\{\mu\left(\nabla \mathbf{u}+\nabla \mathbf{u}^{T}\right)\right\}+\mathbf{F}+\rho \mathbf{g}, \\
& \nabla \cdot \mathbf{u}=\mathbf{0} .
\end{aligned}
$$

To achieve a fully developed state on a high-resolution grid system, the grid points are moved with the same velocity as the tip of the drop $\mathbf{u}_{g}$. The advection velocity in Eq. (7) is therefore presented as the relative velocity following Rosenfeld and Kwak ${ }^{(19)}$. Equations (7) and (8) are discretized in space by a second-order central finite difference method. A secondorder Adams-Bashforth method is applied to advance the advection term through time, and a second-order Crank-Nicolson scheme is applied for the viscous term. Pressure is linked to Eq. (8) by the SMAC algorithm, and the Poisson equation for pressure correction is solved by a SOR method. 


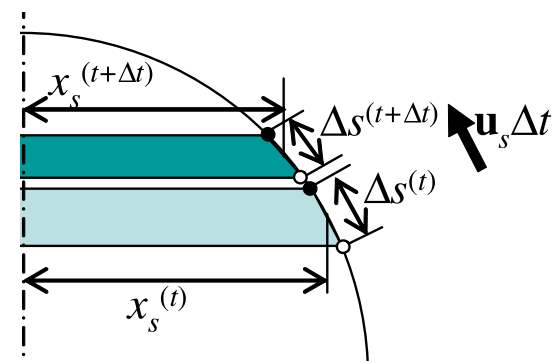

Fig. 1 Advection of a front element. Surfactant on the element is also advected by the front movement. The concentration changes with changes of the area of the element.

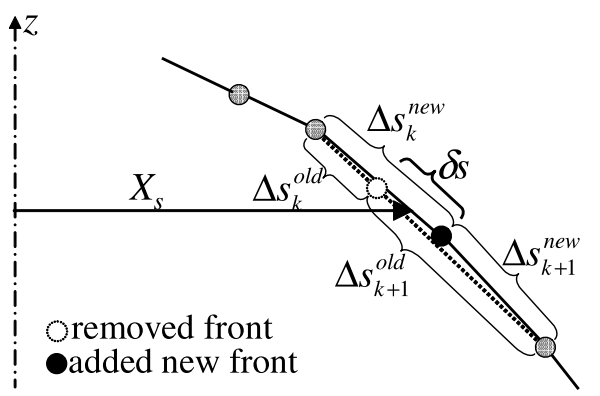

Fig. 2 Change of front element length during the reconstruction of front elements. If the length of an element $k$ becomes shorter than the deletion threshold, then the marker $\circ$ is removed. If the combined length of adjacent elements $(k$ and $k+1)$ exceeds the addition threshold, then the marker $\bullet$ is added. Thus, the length of front element $k$ is increased by $\delta s$ and that of $k+1$ is decreased by $\delta s$.

\subsection{Transport of surfactant}

For the surfactant concentration $\Gamma$ at the interface, the advection-diffusion equation is considered, neglecting adsorption and desorption. The basic equation is derived by Stone ${ }^{(20)}$ as:

$$
\frac{\partial \Gamma}{\partial t}+\nabla_{s} \cdot(\mathbf{u} \Gamma)=D_{s} \nabla_{s}^{2} \Gamma,
$$

where $\nabla_{s}$ is the surface gradient operator. $D_{s}$ is the coefficient of diffusion in the interface, and its unit is $\mathrm{m}^{2} / \mathrm{s}$. In our calculation, the left-hand side representing advection is approximated not by finite difference but by Lagrangian movement of the front elements. The amount of substance rather than its concentration is stored for each front element, so that surfactant advection is automatically represented by the movement of each front element. These movements are represented by the movement of individual front points (corresponding to end points of line segments), so the front element's length is automatically changed by the movement of the front points. A change in concentration caused by the area change is, therefore, also automatically represented by the change of front element's length. For example, in the case shown in Fig. 1, the update of the concentration for advection is calculated by the following conservative relation:

$$
2 \pi x_{s}^{(t+\Delta t)} \Delta s^{(t+\Delta t)} \Gamma^{(t+\Delta t)}=2 \pi x_{s}^{(t)} \Delta s^{(t)} \Gamma^{(t)},
$$

where $x_{s}$ is the radial position of the center of the front element and $\Delta s$ is the length of the element. Superscripts indicate time steps.

As the front points move individually, the elements may become extremely short or long. To avoid the difference of resolution, front points are added and deleted, as mentioned in $\S 3.1$. We propose a scheme that conserves the total amount of surfactant during this process. For example, Fig. 2 shows the front elements $\Delta s_{k}^{\text {old }}$ (corresponding to $\Delta s^{(t+\Delta t)}$ in Fig. 1) before and after a deletion and an addition. First, the marker point $\circ$ is deleted because the length 
of the element $\Delta s_{k}^{\text {old }}$ is shorter than a threshold $T_{d e l}$. Then, the new length of the element $\Delta s_{k}^{\text {old }}+\Delta s_{k+1}^{\text {old }}$ becomes longer than another threshold $T_{\text {add }}$. Subsequently, the new marker point $\bullet$ is added. At that time, the surfactant is redistributed so that the amount of substance contained in the elements with the same area is conserved: in Fig. 2, attention is paid to the region $\delta s$, which changes the element belonging to it. The amount of surfactant contained in the new element $\Delta s_{k}^{\text {new }}$ is given by the following:

$$
2 \pi x_{s k}^{\text {new }} \Delta s_{k}^{\text {new }} \Gamma_{k}^{\text {new }}=2 \pi x_{s k}^{\text {old }} \Delta s_{k}^{\text {old }} \Gamma_{k}^{\text {old }}+2 \pi X_{s} \delta s \Gamma_{k+1}^{\text {old }},
$$

where the $x$-coordinate of the center of the element $\delta s$ is $X_{s}$, that of $\Delta s_{k}^{\text {new }}$ is $x_{s k}^{\text {new }}$, and so on. The amount of surfactant in the element $\Delta s_{k+1}^{\text {new }}$ is decreased by the same amount as the increase in Eq. (11). By such a series of calculations, surfactant advection is completed while completely conserving the total amount of surfactant.

The diffusion term for surfactant transport is treated after the advection stage. The concentration after advection (presented by the superscript*) is discretized on the front element spatially using a central finite difference:

$$
\frac{\Gamma^{(n+1)}-\Gamma^{*}}{\Delta t}=D_{s} \frac{1}{x} \frac{\partial}{\partial s}\left(x \frac{\partial \Gamma}{\partial s}\right),
$$

where $s$ is the arc length coordinate along the interface. For the time progression of advection (corresponding to front movement), a second-order Adams-Bashforth method is applied, and a second-order Crank-Nicolson scheme is applied for the diffusion term. Algebraic equation system for the diffusion term is solved by tridiagonal matrix inversion.

The relation between interfacial tension and interfacial concentration of surfactant is given by a Langmuir model, following Takagi et al. ${ }^{(6)}$ :

$$
\sigma=\sigma_{0}\{1+\alpha \ln (1-\Gamma)\}
$$

where $\sigma_{0}$ is the interfacial tension for the clean interface. The interfacial concentration is made dimensionless by dividing by the maximum interfacial concentration $\Gamma_{\infty}$. The damping coefficient $\alpha$ is represented by the Marangoni number $M a=R_{G} T \Gamma_{\infty} /\left(\mu U_{d}\right)$ and the capillary number $C a=\mu U_{d} / \sigma_{0}$, as $\alpha=M a C a$, where $R_{G}$ is the gas constant and $T$ is absolute temperature.

In our simulation, drops are freely falling, so the terminal velocity $U_{d}$ cannot be given as a preset condition. We therefore set $\alpha$ as one of the preset conditions. If desorption is neglected, surfactant may be accumulated in the wake region without limit, and the maximum interface concentration cannot be defined. If the dimensionless concentration exceeds one, Eq. (13) cannot be evaluated. However, within the range of our simulation conditions, because the Marangoni effect suppresses advection through Eq. (13), the dimensionless concentration never exceeded one.

In this study, the force exerted on a front element by interfacial tension is given by the difference of tangential tension and the effect of curvature in the circumferential direction:

$$
\mathbf{f}_{s}\left(\mathbf{x}_{s}\right) \Delta s=(\sigma \mathbf{t})^{+}-(\sigma \mathbf{t})^{-}+\sigma^{m i d} \frac{n_{x}}{x_{s}} \mathbf{n} \Delta s
$$

where superscripts,+- , and mid indicate the ends and the center of the front element, respectively. $\mathbf{n}=\left(n_{x}, n_{z}\right)$ is the unit normal and $\mathbf{t}$ is the unit tangent. By Eq. (14), the interfacial tension force can be accurately presented and the spatial change of $\sigma$ is naturally represented.

Table 1 Physical properties of fluids.

\begin{tabular}{lll}
\hline & Silicone oil & Water \\
\hline Density $\rho\left[\mathrm{kg} / \mathrm{m}^{3}\right]$ & 997.1 & 965.0 \\
Dynamic viscosity $\mu[\mathrm{Pa} \mathrm{s}]$ & $8.904 \times 10^{-4}$ & $9.650 \times 10^{-2}$ \\
Interfacial tension $\sigma_{0}[\mathrm{~N} / \mathrm{m}]$ & \multicolumn{2}{c}{$5.106 \times 10^{-2}$} \\
\hline
\end{tabular}


Table 2 Conditions of grid resolution test.

\begin{tabular}{lll}
\hline Case & Number of grid points $\left(N_{x} \times N_{z}\right)$ & $\Delta x / d_{d}$ \\
\hline A & $31 \times 124$ & 0.032 \\
B & $62 \times 248$ & 0.016 \\
C & $124 \times 496$ & 0.008 \\
\hline
\end{tabular}

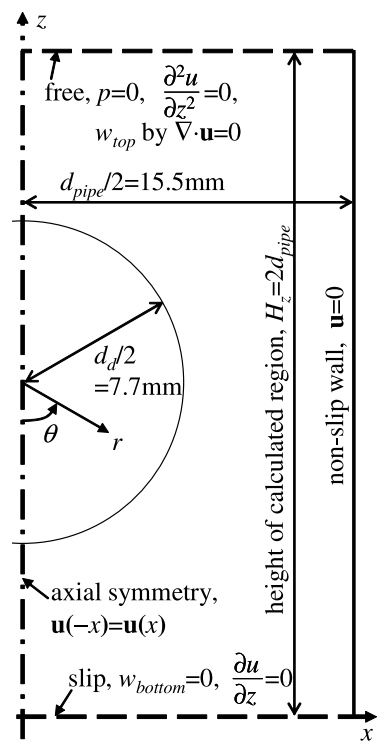

Fig. 3 Computational domain and boundary conditions.

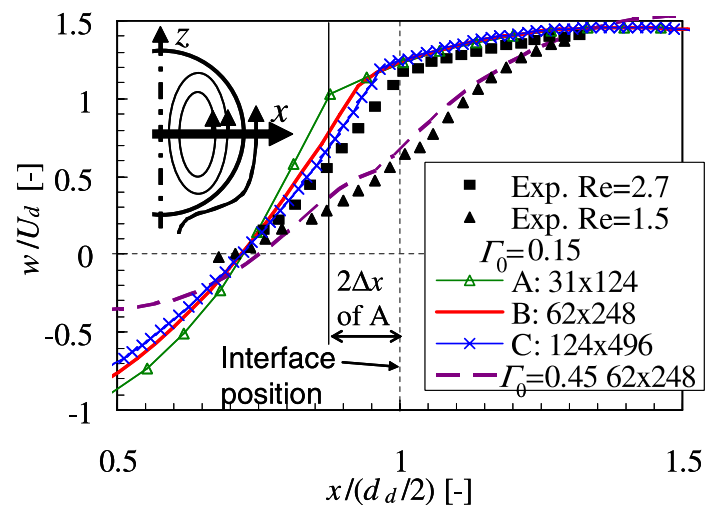

Fig. 4 Distribution of vertical velocity component on the horizontal line through the drop center.

\section{Results and discussion}

\subsection{Simulation conditions}

All the calculations begin from a condition in which both phases are stationary, then the flow field is developed by the freely falling drop. All the results shown in this paper are for the fully developed state. The fully developed states were confirmed by temporal constancy of falling velocity and that of velocity profile. Temporal change of marker distribution does not affect the states. Boundary conditions are shown in Fig. 3. The calculation domain moves with the drop, as mentioned previously. $\theta=0$ on the polar coordinates indicates the bottom of the drop. The vertical length of the computational domain $H_{z}$ was checked by using the condition arising large wake vortex. The differences between cases of $H_{z}=d_{\text {pipe }}, 2 d_{\text {pipe }}, 4 d_{\text {pipe }}, 8 d_{\text {pipe }}$ were little, although the terminal velocity in case of $H_{z}=0.74 d_{\text {pipe }}$ was $1 \%$ larger than that of other cases, where $d_{\text {pipe }}$ is the diameter of the pipe. Then, $H_{z}=2 d_{\text {pipe }}$ was chosen in simulations presented in this paper. 
(a)

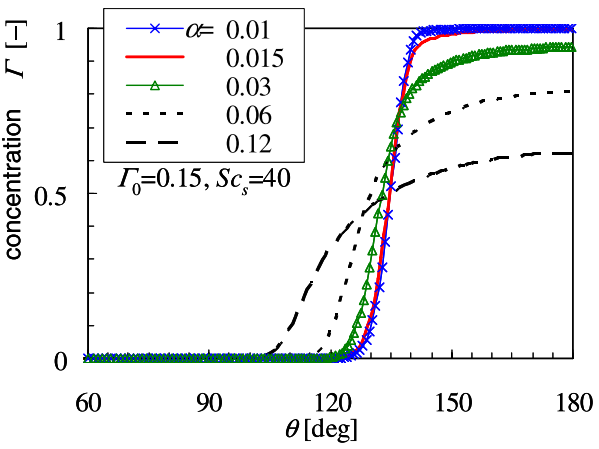

(c)

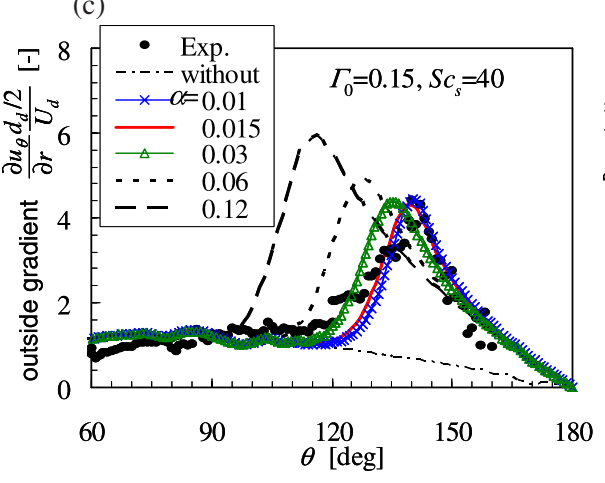

(b)

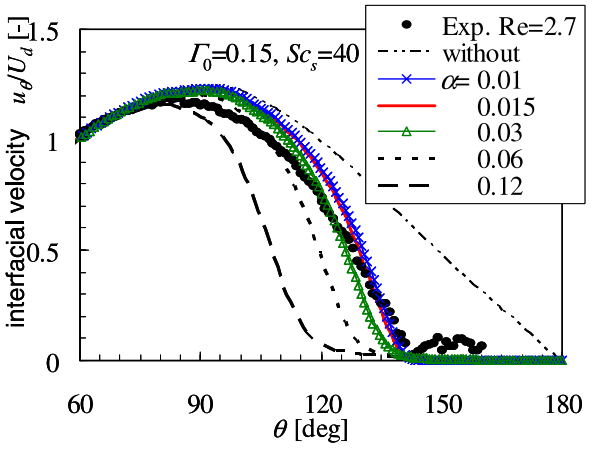

(d)

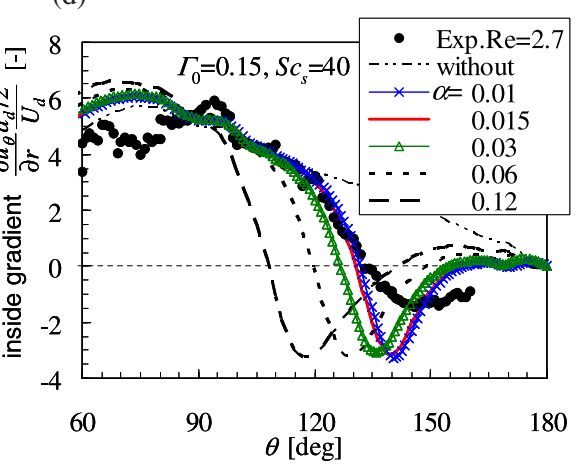

Fig. 5 Effect of the damping coefficient of interfacial tension $\alpha$. (a) concentration distribution, (b) interfacial velocity distribution, (c) radial gradient of tangential velocity outside the interface, (d) radial gradient of tangential velocity inside the interface.

First, the effect of spatial resolution of the fixed grid system was evaluated. The three conditions of spatial resolution shown in Table 2 were tested using appropriate parameters that are discussed in detail later $\left(\Gamma_{0}=0.15, \alpha=0.015, S c_{s}=40\right)$. The grid spacing in the vertical direction was the same as in the radial direction, $\Delta z=\Delta x$. Figure 4 shows the velocity distribution of vertical component on the horizontal line through the drop center. Because the simulated drop is approximately spherical, one on the horizontal axis corresponds to the interface position. Velocities are represented as relative to the drop. At the interface, the salient point is shifted into the low-viscosity fluid within the width of the distribution function, as mentioned in Ref. (21), so the substantial interface position in the low-resolution case A differs greatly from the experimental measurement. The results in case B show some difference from the experimental values. The higher-resolution calculation case $\mathrm{C}$ show improvement in half grid size of $\mathrm{B}$, however, the improvement is much smaller than the length scale of the flow field. In the same figure, results for the other concentration case $\Gamma_{0}=0.45$ on the B grid system are also shown. Comparison of these calculated results with the more contaminated experimental results $(R e=1.5)$ shows very good agreement. Thus, we used the grid system of case B for all subsequent simulations. The time increment was selected as $\Delta t=2.5 \times 10^{-4}$ $\mathrm{s}$ by the criteria of CFL number $<0.1$ and of iteration count for viscous term $<25$.

To evaluate the velocity gradient at the interface inside the drop, taking the salient points into account, the gradient was calculated by a one-sided finite difference at the point 2 grid units from the interface. On the other hand, the experimentally measured data were redistributed on the grid system with $\Delta x / d_{d}=0.013$, and the gradient was obtained by the analytical derivative of a fitted curve of the velocity profile.

\subsection{Effect of the damping coefficient of interfacial tension}

Figure 5 shows the effect of the damping coefficient $\alpha$ on (a) the concentration profile, (b) 
(a)

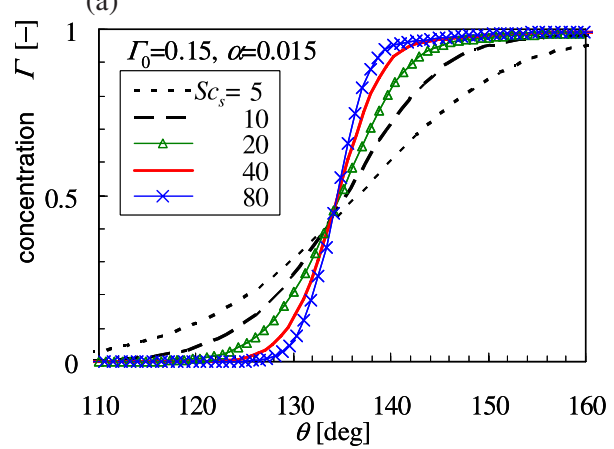

(c)

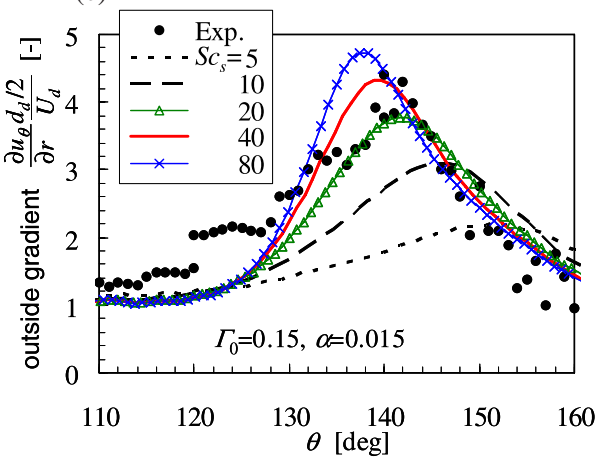

(b)

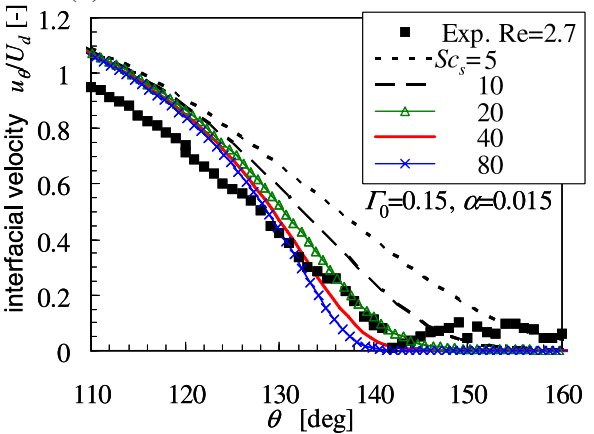

(d)

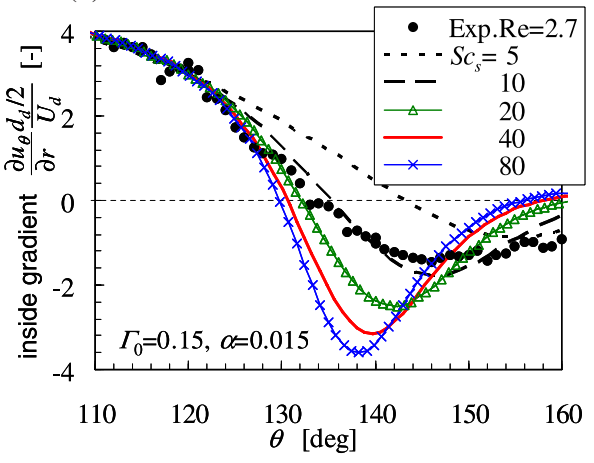

Fig. 6 Effect of Schmidt number $S c_{s}$. (a) Concentration distribution, (b) interfacial velocity distribution, (c) radial gradient of tangential velocity outside the interface, (d) radial gradient of tangential velocity inside the interface.

the tangential velocity profile, and (c), (d) the radial gradient of tangential velocity outside and inside the drop. From Fig. 5(a), we can see that $\alpha$ changes the region containing the surfactant. The corresponding points in (a)-(d) are as follows. The tangential velocity changes strongly at the position at which the concentration begins to increase. Both the outside and the inside velocity gradients have peaks at the position at which the interface velocity drops to zero. An increase in $\alpha$ makes those positions (concentration increasing point, velocity decreasing point, and peak point of velocity gradient) move forward.

\subsection{Effect of diffusion coefficient}

Figure 6 shows the effect of Schmidt number $S c_{s}$ on the same quantities as those in Fig. 5. In this paper, variation of $S c_{s}$ corresponds directly to variation of the interfacial diffusion coefficient $D_{s}$, because the viscosity is constant. With high $S c_{s}$, advection transports surfactant one-sidedly to the rear, so the rear part of the drop has a higher concentration. At the same time, the boundary of the high-concentration region becomes sharp. The main effect of $S c_{s}$ on the concentration profile is not to change the inflection point but to change the tangential gradient of the profile.

As shown in Fig. 5, the velocity changes strongly at the point where the concentration starts to increase for large $\alpha$. On the other hand, for small $\alpha$, as shown in Fig. 6, even though strong diffusion makes the concentration's point of increase move forward, the velocity profile changes little because of the small gradient of interfacial tension. As shown in Fig. 6(a) and (b), the velocity changes quickly at the point where the concentration has a steep gradient (around $\theta=130^{\circ}$ ). As $S c_{s}$ increases, the region in which the concentration changes becomes narrower and the velocity changes sharply within that region. Following those changes, when the spatial gradients of velocity become large, the radial gradients (Fig. 5(c) and (d)) also show intense peaks around the region of increasing $S c_{s}$. 
(a)

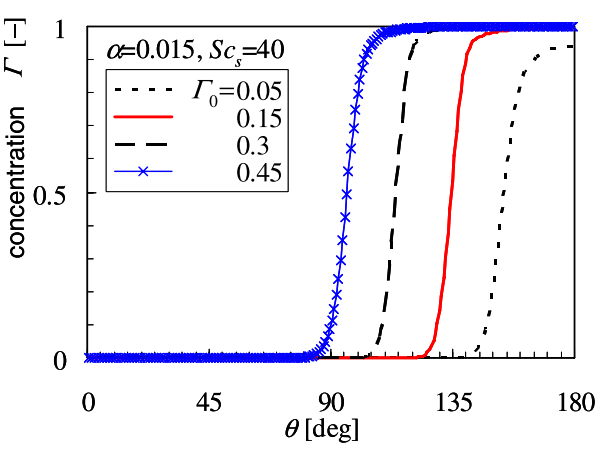

(c)

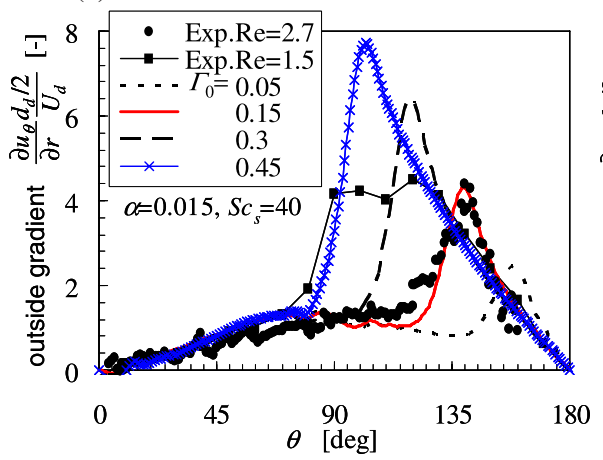

(b)

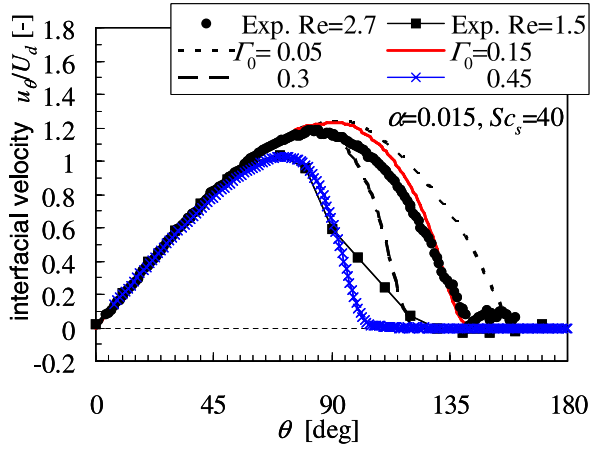

(d)

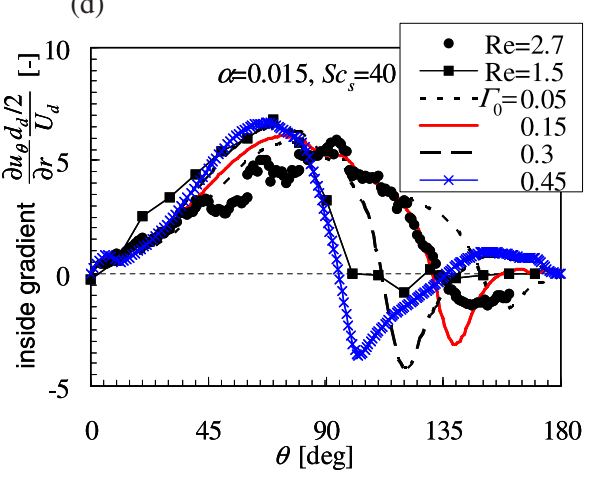

Fig. 7 Effect of mean concentration of surfactant $\Gamma_{0}$. (a) Concentration distribution, (b) interfacial velocity distribution, (c) radial gradient of tangential velocity outside the interface, (d) radial gradient of tangential velocity inside the interface.

\subsection{Effect of total amount of surfactant}

Figure 7 shows the effect of the mean concentration $\Gamma_{0}$, which corresponds to the total amount of adsorbed surfactant. In our simulation conditions of low diffusion coefficient, the concentration at the rear part approaches the maximum value. The effect of $\Gamma_{0}$ thus appears as changes in the increasing points of concentration, as shown in Fig. 7(a). A change of tangential gradient of the concentration causes a change in the velocity's decreasing points, as shown in Fig. 7(b). Because the velocity profile outside the drop varies greatly with $\theta$, the radial velocity gradient changes both its peak position and its peak value, as shown in Fig. 7(c).

By comparing some other calculations with various $\Gamma_{0}, \alpha$ and $S c_{s}$, the condition at which the calculated results agree with the experimental value for $R e=2.7$ is when $\Gamma_{0}=0.15$, $\alpha=0.015, S c_{s}=40$. The interfacial velocity under these conditions, shown in Fig. 7(b), shows good agreement for the edge location of the stagnant cap, while the peak velocity shows a small difference. However, the peak location of the radial velocity gradient and the peak value outside the drop agree very well with the experimental values, except for the peak of the inside gradient. Concerning the gradient inside the drop, the experimental measurement does not have high reliability because of the low tracer concentration near the separation point, and the one-fluid model simulation has the defect of velocity shift into low viscosity as mentioned before. Therefore, we can compare only the rough tendencies for the inside gradient. Furthermore, comparisons of calculated streamlines with experimental path lines are shown in Fig. 8. Reynolds numbers based on the terminal velocity are also shown in the caption. It was found that the calculated result without surfactant differs from the experimental observation. For $\Gamma_{0}=0.15$, the dead water region at the rear of the drop, flow separation of the outside wake, inner circulation, and other variables in the experimental observation for $R e=2.7$ are reproduced faithfully in the present simulation.

If the properties of surfactants are the same through a series of experiments, we can de- 
(a)

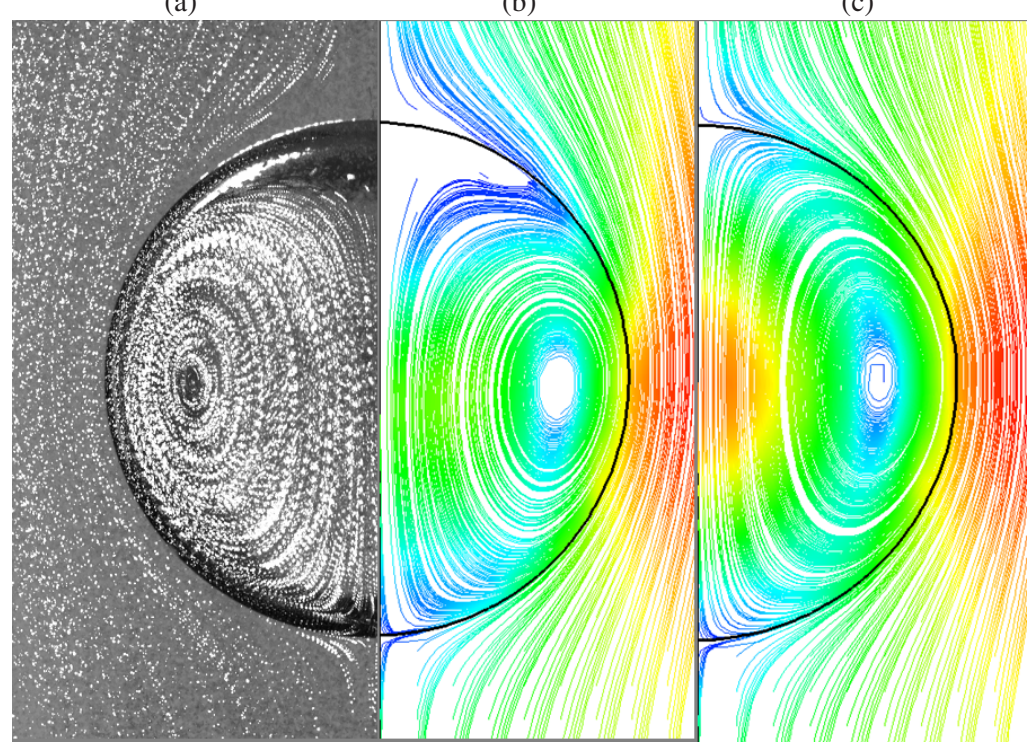

(d)

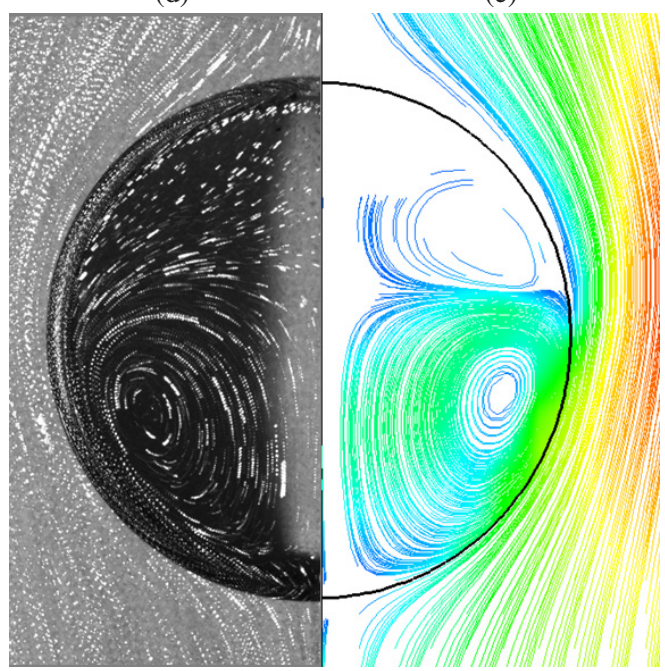

Fig. 8 Calculated streamlines $\left(\alpha=0.015, S c_{s}=40\right)$ and experimental path lines. (a) experiment $(R e=2.7)$, (b) $\Gamma_{0}=0.15(R e=2.7)$, (c) without surfactant $(R e=3.1),(\mathrm{d})$ experiment $(R e=1.5),(\mathrm{e}) \Gamma_{0}=0.45(R e=1.6)$.

duce that the difference in the experimentally observed flow field for $R e=1.5$ on the same experimental facility as that for $R e=2.7$ is caused only by the difference in the total amount of surfactant. Comparing the calculated result of $\Gamma_{0}=0.45$ with fixed $\alpha$ and $S c_{s}$ with the experimental results for $R e=1.5$, the flow field and Reynolds number (corresponding to the terminal velocity) in Fig. 8(e) show very good agreement with those in Fig. 8(d). The interfacial velocity shown in Fig. 7(b) also shows good agreement except at the edge of the stagnant cap (around $\theta=110^{\circ}$ ), where the flow separates and varies strongly; however, the measurement resolution is not high enough at this point, because of the low tracer concentration. The calculated radial gradients shown in Fig. 7(c) and (d) show a large peak at the separation point, while the peaks of experimental results are cut. The evidence supports the supposition of low measurement resolution. Except for the separation point, the radial gradients of the present simulation agree very well with the experimental results as shown in Fig. 7(c) and (d).

Summarizing the above results, which show very good agreement with experimental measurements, we can conclude that the present simulation model reasonably represents the momentum balance of a falling drop in the fully developed state. However, the range of appli- 
cability may be limited, because of neglecting the adsorption/desorption process. Discussion of these limits is a subject for a future study.

\section{Summary}

We proposed the front-tracking method combined with a solver of surfactant transport on the interface. A concrete process of the advection-diffusion calculation on the explicitly represented interfaces was presented, and a fully conserving scheme was proposed. We performed simulations of a water drop sinking in a silicone oil while taking into account the Marangoni effect of the concentration gradient. Three parameters-the damping coefficient of interfacial tension, the interfacial diffusion coefficient, and the total amount of surfactantwere evaluated, and the tendencies of interface concentration, velocity, and velocity gradient were clarified. By the adjustment of these three parameters, the present simulation can reproduce experimentally observed drops accurately. In particular, the stagnant cap size, the location of the separation point, the steep peak of the velocity gradient and the terminal velocity were predicted correctly. We can expect our simulations to estimate the conditions of surfactant on the interfaces.

\section{Acknowledgments}

This work was partly supported by MEXT.KAKENHI (17760149) and (19760122).

\section{References}

( 1 ) Levich, V.G., Physicochemical Hydrodynamics, (1962), Prentice-Hall.

( 2 ) Yamauchi, M., Ozawa, M. and Uemura, T., PTV Measurement in a Region Close to the Interface of a Water Drop in Oil, Japanese Journal of Multiphase Flow, Vol. 14, No. 4, (2000), pp. 466-472 (in Japanese).

( 3 ) Uemura, T. and Yamauchi, M., Shearing Conditions on the Interface of a Spherical Water Drop Sinking in Silicone Oil, KSME International Journal, Vol. 15, No. 12, (2001), pp. 1845-1852.

( 4 ) Bel Fdhila, R. and Duineveld, P.C., The Effect of Surfactant on the Rise of a Spherical Bubble at High Reynolds and Peclet Numbers, Physics of Fluids, Vol. 8, No. 2, (1996), pp. 310-321.

( 5 ) Cuenot, B., Magnaudet, J. and Spennato, B., The Effects of Slightly Soluble Surfactants on the Flow around a Spherical Bubble, Journal of Fluid Mechanics, Vol. 339, (1997), pp. 25-53.

( 6 ) Takagi, S., Yamamoto, A. and Matsumoto, Y., Numerical Analysis of a Rising Bubble in a Contaminated Liquid, Proceedings of the 3rd ASME/JSME Joint Fluids Engineering Conference, (1999), FEDSM99-7102 (CD-ROM).

( 7 ) Liao, Y. and McLaughlin, J.B., Bubble Motion in Aqueous Surfactant Solutions, Journal of Colloid and Interface Science, Vol. 224, (2000), pp. 297-310.

( 8 ) Takagi, S. and Matsumoto, Y., Contaminant Effect on the Motion of a Rising Bubble, Proceedings of ASME 2000 Fluids Engineering Division Summer Meeting, (2000), FEDSM2000-11269 (CD-ROM).

( 9 ) Hirt, C.W. and Nichols, B.D., Volume of Fluid (VOF) Method for the Dynamics of Free Boundaries, Journal of Computational Physics, Vol. 39, (1981), pp. 201-225.

(10) Unverdi, S.O. and Tryggvason, G., A Front-Tracking Method for Viscous, Incompressible, Multi-Fluid Flows, Journal of Computational Physics, Vol. 100, (1992), pp. 25-37.

(11) Sussman, M., Smereka, P. and Osher, S., A Level Set Approach for Computing Solutions to Incompressible Two-Phase Flow, Journal of Computational Physics, Vol. 114, (1994), pp. 146-159.

(12) Yabe, T., Xiao, F. and Utsumi, T., The Constrained Interpolation Profile Method for Multiphase Analysis, Journal of Computational Physics, Vol. 169, (2001), pp. 556-593.

(13) James, A.J. and Lowengrub, J., Surfactant-Conserving Volume-of-Fluid Method for In- 
terfacial Flows with Insoluble Surfactant, Journal of Computational Physics, Vol. 201, (2004), pp. 685-722.

(14) Xu, J.J. and Zhao, H.K., An Eulerian Formulation for Solving Partial Differential Equations along a Moving Interface, Journal of Scientific Computing, Vol. 19, (2003), pp. 573-594.

(15) Jan, Y. and Tryggvason, G., Computational Studies of Contaminated Bubbles, Proceedings of the Dynamics of Bubbles and Vortices near a Free surface, ASME/AMD, Vol. 119, (1991), pp. 59-64.

(16) Homma, S., Koga, J., Matsumoto, S. and Tryggvason, G., Solutal-Capillary Motion of a Liquid Jet and its Breakup into Drops, Proceedings of the 3rd ASME/JSME Joint Fluids Engineering Conference, (1999), FEDSM99-7114 (CD-ROM).

(17) Tryggvason, G., Bunner, B., Esmaeeli, A., Juric, D., Al-Rawahi, N., Tauber, W., Han, J., Nas, S. and Jan, Y.-J., A Front-Tracking Method for the Computations of Multiphase Flow, Journal of Computational Physics, Vol. 169, (2001), pp. 708-759.

(18) Peskin, C.S., Numerical Analysis of Blood Flow in the Heart, Journal of Computational Physics, Vol. 25, (1977), pp. 220-252.

(19) Rosenfeld, M. and Kwak, D., Time-dependent Solutions of Viscous Incompressible Flows in Moving Coordinates, International Journal for Numerical Methods in Fluids, Vol. 13, (1991), pp. 1311-1328.

(20) Stone, H.A., A Simple Derivation of the Time-Dependent Convective-Diffusion Equation for Surfactant Transport along a Deforming Interface, Physics of Fluids Vol. A2, No. 1, (1990), pp. 111-112.

(21) Ferziger, J.H., Interfacial Transfer in Tryggvason's Method, International Journal for Numerical Methods in Fluids, Vol. 41, (2003), pp. 551-560. 